\section{Impacto de una capacitación en trastornos depresivos para médicos generales de atención primaria en salud. Resultados cualitativos}

\author{
GABRIELA HUEPE O. ${ }^{1, \mathrm{a}}$, MARCELO CÁRCAMO Q. ${ }^{1, \mathrm{~b}}$, JULIA ACUÑA R. ${ }^{1}$, \\ ALBERTO BOTTO V. ${ }^{1}$, JUAN PABLO JIMÉNEZ ${ }^{1}$
}

\section{Qualitative impact of a training program on depressive disorders for primary care physicians}

Background: There are suggestions that, despite training efforts for Primary Health Care physicians, difficulties in making accurate diagnoses and timely treatments persist. Aim: To evaluate the impact of a new training model in Depressive disorders integrated with Mindful Practice, on the diagnostic and therapeutic skills of primary care general physicians. Material and Methods: $A$ voluntary sample of 56 general physicians was studied. The design of this study was experimental and randomized, with two groups attending theoretical sessions followed by differing workshops that were carried out in a parallel fashion. The Quantitative phase of this study considered measuring the impact of training in physician's skills. The Qualitative phase included nine semi-structured interviews and Qualitative Content Analysis. This paper reports the results of the interviews. Results: As a consequence of training sessions, physicians learned that above the mental health problems, other issues such as self-efficacy and self-confidence are important for the management of depressive patients. Conclusions: This qualitative study shows that physicians are obtaining significant benefits from their training sessions.

(Rev Med Chile 2015; 143: 795-800)

Key words: Clinical competence; Depressive disorder; Primary Health Care; Qualitative Research.
'Departamento de Psiquiatría y Salud Mental Oriente, Facultad de Medicina, Universidad de Chile. Santiago, Chile. aLicenciada en Sociología, Magíster (c) en Métodos para la Investigación Social.

'Psicólogo, Postítulo en Psicoterapia Focal Psicoanalítica, Magíster en Psicoterapia, Doctorado (c) en Investigación en Psicoterapia.

\section{Apoyo Financiero:}

Proyecto FONIS SA09I20014, financiado por el Fondo Nacional de Investigación y Desarrollo en Salud (FONIS), CONICYT-MINSAL. Convocatoria IX Concurso, 2009. Los autores no han recibido apoyo de ningún tipo para escribir este artículo, ni han tenido influencia en el diseño ni contenido del mismo.

Recibido el 15 de octubre de 2014, aceptado el 15 de abril de 2015.

\section{Correspondencia a:}

Gabriela Huepe O.

Av. Salvador 486, Providencia,

Santiago de Chile.

Cel: 5-6475208

gabrielahuepe@med.uchile.cl
M últiples estudios han demostrado una alta prevalencia anual de trastornos mentales en Chile, ubicándose las tasas en los rangos más altos ${ }^{1}$. Un reciente metanálisis, que evaluó estudios sobre la precisión de profesionales médicos sin especialización en psiquiatría para reconocer la depresión, en casi sesenta mil pacientes adultos de atención primaria y hospitalizados, concluyó que los profesionales presentan una capacidad limitada para diagnosticarla ${ }^{2}$.

Esto ha justificado en Chile la implementación del Programa Nacional para la Detección y Tratamiento de la Depresión y su posterior incorpora- ción dentro de las patologías GES. Sin embargo, múltiples investigaciones han demostrado que, a pesar de los esfuerzos desplegados en la capacitación en Atención Primaria de Salud (APS), persisten las dificultades para establecer diagnósticos oportunos y tratamientos adecuados ${ }^{3}$.

Un estudio chileno ${ }^{4}$, mostró carencias en la evaluación del programa para la detección, diagnóstico y tratamiento integral de la depresión. Posteriormente, Vicente et al. en 2007, reconocieron que una capacitación meramente cognitiva en depresión para médicos generales, tuvo limitado impacto en la práctica clínica donde el cambio en 
conocimientos y actitudes fue escaso. Concluyen que es más prometedor incluir una capacitación en otras fortalezas a través del entrenamiento en actitudes y destrezas clínicas específicas.

Existe consenso en que el diagnóstico en psiquiatría trasciende la mera descripción de síntomas, pasando por la auto observación del vínculo emocional que el médico establece con su usuario ${ }^{5}$, lo que ha sido incorporado en la formación médica como Práctica Reflexiva ${ }^{6}$. La Práctica Reflexiva implica atender tanto a las acciones observables como a los estados emocionales, a través de sus cuatro atributos: observación atenta, curiosidad crítica, flexibilidad informada y presencia. Esto resulta central en un darse-cuenta, comprender y responder a las necesidades de los usuarios.

El presente artículo surge en relación con el proyecto de investigación del Fondo Nacional de Investigación y Desarrollo en Salud (FONIS) (SA09I20014), cuyo propósito fue evaluar el impacto de un nuevo modelo de capacitación en trastornos depresivos integrado con Práctica Reflexiva, sobre las habilidades diagnósticas y terapéuticas del médico general de APS. El estudio fue aprobado por el Comité de Ética de Investigación con Seres Humanos de la Facultad de Medicina de la Universidad de Chile. El diseño fue experimental randomizado, con dos grupos experimentales sometidos a 3 sesiones conjuntas de clases teóricas y dos tipos distintos de Taller de 10 sesiones realizados en forma paralela (Tabla 1).

La muestra fue de tipo voluntaria, a través de la difusión (afiches, cartas, etc.) del estudio y la capacitación dirigida a la población de médicos generales de APS (Consultorios) de Servicios de Salud de la Región Metropolitana. El total de interesados fue de 76 médicos y 56 fueron seleccionados para participar en el estudio, luego de cumplir con los siguientes criterios de inclusión: tener una jornada laboral de $22 \mathrm{~h}$ semanal o más en los consultorios participantes y un mínimo de 8 meses de experiencia en APS.

La fase cuantitativa del estudio contempló la medición del impacto de las capacitaciones en las habilidades diagnósticas y terapéuticas de los médicos, en tres momentos: basal, al término de la capacitación y a los tres meses.

La investigación tuvo una fase cualitativa, fundamentada en el interés de acceder desde la visión de los propios médicos participantes, a los elementos de la capacitación que consideraron más relevantes e impactantes en su práctica médica y que en consecuencia, vinculan con el desarrollo de sus habilidades diagnósticas y terapéuticas de la depresión en APS. El presente artículo muestra los resultados de esta segunda fase cualitativa.

\section{Método}

En el marco del estudio cuyo diseño fue experimental randomizado, se incorporó una estrategia de complementación de métodos ${ }^{7}$ a través de la utilización de técnicas de recolección y análisis de información cualitativos con el fin de acceder y describir la visión que tienen los participantes de la capacitación sobre el impacto de la ésta en sus habilidades clínicas.

La muestra en esta fase cualitativa se definió en base a los resultados de la segunda medición cuantitativa, considerando como criterio de selección en cada grupo de estudio, el aumento y disminución en los puntajes de conocimientos teóricos, reconocimiento y clasificación de síntomas en el diagnóstico de la depresión. Además, se incluyó a dos participantes cuyo desempeño en la capacitación fue favorable de acuerdo a la percepción subjetiva de los capacitadores (Tabla 2). El total de entrevistados fueron 9 médicos, 6 chilenos y 3 extranjeros, 5 hombres y 4 mujeres y todos con jornada completa en consultorios. La edad varía

\section{Tabla 1. Metodología Talleres Práctico y Reflexivo}

\section{Taller Práctico}

Metodología experiencial y participativa, basada en revisión de casos clínicos videados y casos aportados por los propios participantes

\section{Taller Reflexivo}

Metodología activa-participativa, en parejas o grupos pequeños, con ejercitación de "práctica informal" principalmente mediante estímulos audio-visuales y ejercitación de "práctica formal" de tipo individual mediante la técnica de meditación mindfulness 
Tabla 2. Constitución de la muestra de entrevistados en la fase cualitativa

\begin{tabular}{|c|c|c|c|c|}
\hline \multirow[t]{2}{*}{ Casos } & \multicolumn{2}{|c|}{$\begin{array}{l}\text { Aumenta puntuación } \\
\text { reconocimiento y clasificación } \\
\text { de síntomas }\end{array}$} & \multicolumn{2}{|c|}{$\begin{array}{l}\text { Disminuye puntuación } \\
\text { reconocimiento y clasificación } \\
\text { de síntomas }\end{array}$} \\
\hline & Grupo Práctico & Grupo Reflexivo & Grupo Práctico & Grupo Reflexivo \\
\hline $\begin{array}{l}\text { Aumenta puntuación en } \\
\text { Conocimiento Teórico }\end{array}$ & 1 & 1 & 1 & 1 \\
\hline $\begin{array}{l}\text { Disminuye puntuación en } \\
\text { Conocimiento Teórico }\end{array}$ & 1 & 1 & 1 & No hay casos \\
\hline $\begin{array}{l}\text { Criterio subjetivo } \\
\text { capacitadores }\end{array}$ & 1 & 1 & & \\
\hline
\end{tabular}

entre los 26 y 60 años, con una media de 34,7 años y una mediana de 30 años. El tiempo de experiencia en APS fluctúa entre los 13 y 64 meses, con una mediana de 42 meses y una media de 38,2 meses.

Se realizaron entrevistas semi-estructuradas, en base a una pauta temática que fue discutida y corregida por el equipo de investigación en su conjunto, resguardando dar respuesta al objetivo comprometido. Se aplicó el total de entrevistas en 5 semanas, luego de un mes de haber finalizado la capacitación y un mes antes de la tercera medición cuantitativa. La duración de cada entrevista fue entre 45 a $60 \mathrm{~min}$, las que se registraron en audio y fueron transcritas por una sola persona externa al equipo investigador.

Se realizó Análisis de Contenido, entendido como una "técnica de investigación destinada a formular, a partir de ciertos datos, inferencias reproducibles y válidas que pueden aplicarse a su contexto" $"$. La técnica consiste en clasificar y codificar los diversos elementos de un mensaje en categorías con el fin de hacer aparecer de manera adecuada su sentido, permitiéndonos así verificar la presencia de temas, de palabras o de conceptos en un contenido. Se aplicó un sistema de categorías y codificación construido deductivamente sobre la pauta de entrevista e inductivamente en base a la lectura de las entrevistas, que posteriormente fue sometido a validación inter-codificadores contrastando su grado de concordancia (85\%) para luego ser aplicado al conjunto de entrevistas por parte de un sólo integrante del equipo investigador. Las categorías de análisis resultantes abordaron la valoración y expectativas de la capacitación para su práctica médica, de los contenidos teóricos y de las metodologías de las capacitaciones, así como la percepción de impacto de la capacitación en sus habilidades diagnósticas y terapéuticas.

A continuación se presentan los resultados del análisis del conjunto de entrevistas, enfatizando los tópicos de las capacitaciones y el impacto de la misma en su práctica clínica, más que la pertenencia a un determinado grupo de capacitación o en relación a resultados obtenidos en la medición cuantitativa.

\section{Resultados}

\section{a) Evaluación de la capacitación}

La evaluación general dio cuenta que los entrevistados coinciden en valorar positivamente la capacitación en ambos momentos, módulo teórico y talleres, aunque se otorga mayor valor a los talleres por significarlos como la oportunidad para aplicar los contenidos del módulo teórico (principalmente en el caso del Taller Práctico) y por el aporte a un mayor bienestar personal que impactaría también en lo profesional (principalmente en el caso el Taller Reflexivo).

En la evaluación Módulo Teórico, los entrevistados destacan la calidad y cantidad de los contenidos entregados y su aplicabilidad en la práctica médica cotidiana. Hay coincidencias en solicitar: a) profundizar en diagnóstico diferencial y tratamiento farmacológico, y b) diferenciar mejor los tratamientos en poblaciones diversas, como por ejemplo adultos mayores, embarazadas $\mathrm{y}$ adolescentes.

"Profundizar más en los aspectos de la depresión en las distintas edades. Porque uno ve bastantes 
jóvenes bajo 18 años y con serios problemas en trastornos anímicos, y en ese sentido igual la sintomatología no es la misma [...] También en la parte geriátrica que hay una gran prevalencia e incidencia, pero obviamente son más enmascaradas, son como más... en ese sentido profundizar un poquito más..." (P1_ST-SD).

En cuanto a la evaluación del Taller Reflexivo, hay una positiva valoración que se fundamenta precisamente en aspectos relacionados con la Práctica Reflexiva, como por ejemplo el hecho de haber aprendido a respirar, relajarse y dejar de lado ideas molestas, lo que además se valora por su aplicabilidad a nivel personal y profesional. Un participante destaca este taller como un espacio terapéutico, en el sentido de entenderlo como una oportunidad para compartir experiencias sobre la práctica médica con pares que presentan vivencias similares. Este aprendizaje de la Práctica Reflexiva, es caracterizado como necesario, aludiendo a la formación médica que deja de lado el bienestar del propio médico (autocuidado).

"Súper útil como en lo personal sobre todo, incluso mucho más que en la parte laboral, a ver, no tanto en lo teórico como sí en la forma de ver las cosas que uno está viendo de mirar el trabajo de uno, como muy como terapéutico pa' uno, o sea como, no soy el único que le pasa esto, un poco compartir las experiencias, bajar la angustia frente a algunas cosas que uno no maneja tan bien de repente, como eso (...) Si, o sea sobre todo la parte del mindfulness, eso más personal, en la vida personal" (R9_CS).

En la evaluación del Taller Práctico en cambio, las valoraciones se fundamentan en la entrega de conocimientos teóricos y su aplicación en ejemplos prácticos a través de casos clínicos videados (provistos por el taller) y otros relatados por los propios participantes. No obstante, esperan que los casos clínicos utilizados sean más típicos y representativos de la realidad cotidiana en APS. También destacan el reforzamiento y ejercicio práctico del Examen Mental como una ayuda concreta al diagnóstico, así como la instancia de feedback y conversación grupal de los casos clínicos como parte de la metodología que favorece el aprendizaje.

"Sí sabía [que existía el Examen Mental], pero no sabía cómo hacerlo y realizarlo de forma sistemática; en ese sentido la parte práctica me hizo como sistematizar un poquito los contenidos del examen mental, cómo sacar la información del paciente, que también es súper importante, porque de eso depende el diagnóstico y [...] en ese sentido fue de ultra utilidad" (P1_ST-SD).

Al preguntar por el aporte de los contenidos teóricos a la práctica médica, los médicos formados en el extranjero principalmente, reconocen tener menor manejo en temas de psiquiatría y salud mental, catalogándola como una capacitación complementaria y necesaria para su práctica médica en Chile. Además, la valoración de los diferentes contenidos de la capacitación, depende en alguna medida del grado de interés que el entrevistado tenga en el área de la psiquiatría, pudiéndose requerir más especificidad en el abordaje de los contenidos cuando hay un interés mayor en la disciplina. En general, el aporte visualizado a nivel teórico, se vincula al reforzamiento de conocimientos y la entrega de elementos que facilitaron una mayor estructura o esquemas claramente establecidos (algoritmos) en torno al diagnóstico y tratamiento en trastornos depresivos en APS. Los principales contenidos teóricos valorados positivamente son Diagnóstico Diferencial y Manejo Farmacológico. Menos valorado por un entrevistado, es el contenido sobre Epidemiología de la Depresión.

\section{b) Percepción de Impacto en los $M G$}

Los principales cambios percibidos por los entrevistados en sus habilidades diagnósticas, son: a) Forma menos mecánica de utilizar los criterios del CIE-10 para realizar diagnóstico de depresión; b) Mayor seguridad frente al proceso diagnóstico; c) Mayor capacidad de observación, un estado más alerta y de conexión frente al paciente; d) Mayor indagación, pesquisa de información; e) Mayor empatía con el paciente orientada al diagnóstico; f) Mayor orden, aplicación de esquemas mentales para el diagnóstico, al estilo examen físico; g) Mayor capacidad de auto-observación emocional en relación a las historias de vida de los pacientes; $h$ ) Menor premura en realizar el diagnóstico, dado el conocimiento adquirido y la posibilidad de que los síntomas no correspondan necesariamente a trastornos depresivos.

“... el diagnóstico diferencial pensamos que era depresión y no lo era, llevamos muchos casos a presentarlos y el primer diagnóstico de nosotros era depresión y empezamos a hablar y hablar $y$ efectivamente no era depresión, sino era otra cosa. 
Yo creo que el diagnóstico diferencial nos ayudó, yo creo que ahora van a haber menos depresiones moderadas" (P5_BT-SD).

Los cambios percibidos por los médicos entrevistados en torno a las habilidades de tratamiento, son: a) Mayor amplitud y flexibilidad en el uso del arsenal medicamentoso disponible; b) Mayor indicación a mujeres embarazadas; c) Mayor entrega de información a los pacientes respecto de efectos adversos o psicoeducación, orientada a lograr mayor adherencia al tratamiento; d) Mayor indagación y educación respecto de la prescripción medicamentosa; e) Mejor gestión para el uso racional de los recursos farmacológicos al interior del consultorio.

Respecto de la diferenciación de las etapas del tratamiento, algunos médicos declaran un conocimiento superficial o desconocimiento de ésta hasta el momento de la capacitación. El argumento de "pérdida de miedo en aumentar dosis" se evidencia en algunos casos como el principal cambio en la práctica médica dentro del ámbito de tratamiento. Respecto de la derivación, como otra dimensión de estas habilidades, se observa que en general los entrevistados dan cuenta de menores derivaciones luego de la capacitación, a excepción de un sólo entrevistado que declara haber aumentado las derivaciones. Todos relatan sentirse más capacitados para realizar un buen diagnóstico como argumento principal para justificar el derivar más o menos a especialistas.

\section{Discusión}

La valoración positiva de la capacitación por parte de los médicos participantes, tanto en el módulo teórico común, así como en los talleres diferenciados (Práctico y Reflexivo), puede constituir un resultado bastante evidente en la medida que la muestra fue constituida por voluntarios, es decir, por médicos que posiblemente reconocen falencias formativas y prácticas, como en el caso de los médico extranjeros, o bien que poseen un interés o sensibilidad particular por la disciplina psiquiátrica o el ámbito de la salud mental.

No obstante, los contenidos detrás de esta favorable valoración nos entregan valiosa información sobre qué aspectos particulares son considerados relevantes para la práctica médica en APS, resaltando aspectos teóricos (diagnóstico diferencial, tratamiento farmacológico para poblaciones di- versas), metodologías de enseñanza-aprendizaje (examen mental, aplicación contenidos en casos clínicos videados) y, en el caso del Taller Reflexivo, aspectos vinculados al bienestar subjetivo de los propios médicos (respirar, relajarse, dejar de lado ideas molestas, espacio terapéutico).

En relación con los contenidos teóricos, cabe plantear la pregunta de si el currículo de pregrado actual considera suficientemente la importancia de la salud mental en APS, en concordancia con las políticas públicas en salud. Una pregunta similar podemos hacer en relación con las competencias, dado que la enseñanza universitaria tradicionalmente se ha centrado en los contenidos teóricos, descuidando los aspectos del "saber hacer". En este sentido la valoración positiva del examen mental, y de la aplicación contenidos en casos clínicos videados, como actividades prácticas, destaca la necesidad de un currículo basado en competencias.

Respecto del Taller Reflexivo, se reafirma lo planteado en otro estudio, en el sentido que cuando la capacitación desarrolla habilidades intra e interpersonales, mejora la capacidad de cuidados centrados en el paciente y los médicos se sienten capaces de atender mejor sus necesidades. Además, el compartir experiencias con colegas reduce el aislamiento profesional ${ }^{10}$. Nuestro estudio destaca otro aporte importante de este taller, cual es el fomento del bienestar subjetivo de los profesionales y la promoción de su capacidad de autocuidado.

La evaluación de competencias en capacitaciones en psiquiatría es un tema en desarrollo. De acuerdo con los propios médicos entrevistados, existen elementos más allá de los conocimientos que son importantes para enfrentar pacientes con problemas de salud mental, como auto eficacia y confianza ${ }^{11}$, los que podemos considerar como un efecto de los talleres implementados.

Los resultados cualitativos de este estudio favorecerán el diseño de futuras capacitaciones a profesionales o estudiantes de pregrado del área de la salud, potenciando así el mejor desarrollo de sus habilidades clínicas.

\section{Referencias}

1. Vicente B, Kohn R, Levav I, Espejo F, Saldivia S, Sartorius $\mathrm{N}$. Trainning primary care physicians in Chile in the diagnosis and treatment of depression. J Affect disord 2007; 98 (1-2): 121. 
2. Cepoiu M, McCusker J, Cole M, Sewitch M, Belzile E, Ciampi A. Recognition of Depression by non-psychiatric Physicians: a systematic literature review and meta-analysis. Journal of General Internal Medicine 2008; 23: 25-2.

3. Levav I, Kohn R, Montoya I, Palacio C, Rozic P, Solano I, et al. Training Latin American Primary care physicians in the WPA module on depression: results of a multicenter trial. Psychol Med 2005; 35 (1): 35-45.

4. Alvarado R, Vega J, Sanhueza G, Muñoz MG. Evaluación del Programa para la Detección, Diagnóstico y Tratamiento Integral de la Depresión en Atención Primaria en Chile. Revista Panamericana de Salud Pública 2005; 18 (4/5): 278-86.

5. Seeling M, Katon W. Gaps in depression care: why Primary Care Physicians should hone their depression screening, diagnosis, and Management skills. Journal of Occupational and Environmental Medicine 2008; 50: 451-8.

6. Epstein R, Quill T, Krasner M, McDonald A. Curricu- lum in Mindful Practice for Students and Residents. Faculty Manual. School of Medicine and Dentistry, University of Rochester 2007 (Documento no publicado).

7. Verd JM, López P. La eficiencia teórica y metodológica de los diseños Multimétodo. EMPIRIA, No 16, julio-diciembre, 2008, pp. 13-42.

8. Krippendorff K. Metodología de análisis de contenido. Teoría y Práctica. Barcelona: Paidós; 1990.

9. Gómez MA. Análisis de contenido cualitativo y cuantitativo: Definición, clasificación y metodología. Revista de Ciencias Humanas 2000; $\mathrm{N}^{\circ} 20$. Colombia.

10. Beckman H, Wendland M, Mooney Ch, Krasner M, Quill Th, Suchman A, et al. The impact of a program in mindful communication on primary care physician. Acad Med 2012; 87: 1-5.

11. Triana C, Olson M, Trevino D. A new paradigma for teaching behaviour change: implications for residency training in family medicine and psychiatry. BCM Medical Education 2012, 12: 64. Disponible en www. biomedcentral.com [Consultado el 2 de abril de 2014]. 